\title{
A Fixed Angle Double Mirror Filter for Preparing a Pink Undulator Beam at the Advanced Photon Source
}

\author{
E. Dufresne, T. Sanchez, T. Nurushev, R. Clarke and S.B. Dierker
}

Dept. of Physics, The University of Michigan Ann Arbor MI 48109-1120

\begin{abstract}
Recent advances in X-ray Photon Correlation Spectroscopy (XPCS) use the full bandwidth of an undulator harmonic in order to maximize the coherent flux for small angle X-ray scattering experiments. X-ray mirrors and filters are typically used to select a given harmonic of the spectrum. At the University of Michigan/Howard University/Lucent Technologies, Bell Labs, Collaborative Access Team (MHATT-CAT) undulator beamline of the Advanced Photon Source, we have designed a fixed-angle Double Mirror Filter which will provide a "pink beam" (i.e., 2-3 \% bandwidth) for XPCS experiments. This device uses two small mirrors which vertically reflect a 0.1 $\mathrm{mm} \times 0.1 \mathrm{~mm}$ white beam in a symmetric geometry. The doubly reflected beam propagates parallel to the incident white beam, but is offset vertically by $35 \mathrm{~mm}$. Using the standard offset of the APS allows one to stop the white beam with a standard APS beam stop. In this report, we will describe our design considerations for this instrument. We also report the results of preliminary tests of the performance. The mirrors preserve the transverse coherence of the source, and filter the undulator spectrum as expected.
\end{abstract}

X-ray Photon Correlation Spectroscopy (XPCS) can often benefit from using the full bandwidth of an undulator harmonic in order to maximize the coherent flux for small-angle X-ray scattering (SAXS) experiments [1]. In a typical set up, a combination of vertical and/or horizontal grazing incidence mirrors is used to filter out the high energies of the undulator spectrum. This provides a "pink" beam, i.e. one whose energy and bandwidth are essentially those of the undulator fundamental. Optimum operation involves tuning the mirror reflection angle so as to place the mirror cut-off energy just above the fundamental energy. This typically places the mirror reflection angle between 5 to $15 \mathrm{mrad}$. It is also desirable to have a substantial offset between the white beam and the pink beam to facilitate shielding from the white beam high energy radiation. The standard monochromatic beam vertical offset at the Advanced Photon Source (APS) is $35 \mathrm{~mm}$. A combination of two vertical reflections serves to preserve the beam direction, which obviates the need for an articulated beamline. Together, these requirements dictate a large 


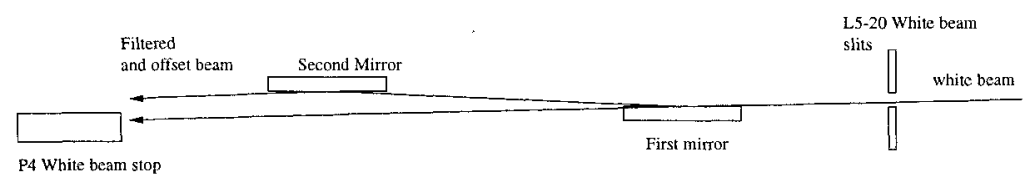

FIGURE 1. The double mirror filter set up.

downstream separation and a large variation in separation, with both on the order of meters, between two vertically reflecting mirrors.

To reduce the complexity and cost of such a design, it is possible to work at a single fixed-angle and still cover most of the energy range typically used in XPCS. This approach is based on the realization that the coherent flux and spectral purity of the resulting pink beam are only weakly dependent on the precise mirror angle. By providing multiple mirror coatings in the form of stripes, it is possible to have a significant range of tunability even at a fixed angle. We present below some design considerations and some preliminary performance of a fixed-angle double mirror filter for an undulator beamline of the APS.

Fig. 1 shows a schematic drawing of the double mirror filter in the beamline. In order to provide "pink" beam at the University of Michigan, Howard University, Lucent Technologies, Bell Labs, Collaborative Access Team (MHATT-CAT) Insertion Device beamline of the APS, we are installing a double-bounce vertically deflecting mirror set up with a $35 \mathrm{~mm}$ offset. Both mirrors are housed in UHV vacuum tanks. The first mirror tank will be placed just before our cryogenically cooled double crystal monochromator (not shown in Fig. 1), while the second tank will be placed approximately $2 \mathrm{~m}$ behind the first mirror tank, just downstream of the monochromator tank. After the second mirror tank, a standard APS beam stop will stop any stray white beam from propagating, while allowing the offset and filtered beam to enter the downstream experimental hutches.

The combination of a $35 \mathrm{~mm}$ offset and a $2 \mathrm{~m}$ mirror separation forces us to work at a fixed mirror angle of approximately $8.75 \mathrm{mrad}$. To extend the cutoff energy of the mirrors at this relatively large angle, we have purchased two small Si flats each coated with a strip of Rh and Pt. Fig. 2 shows the combined reflectivity of the two mirrors as a function of energy. The combined reflectivity is $61 \%$ and $54 \%$ for Pt and $R h$ respectively. The $R h$ and $P t$ strips are useful for energies up to 7.5 and $9 \mathrm{keV}$ respectively. The effect of the reflectivity of two Pt mirrors is also shown in Fig. 2 on an APS white beam simulated by the X-ray utility program XOP [2] with current APS source parameters [3]. The fundamental is at $7.64 \mathrm{keV}$, and the mirror clearly suppresses the second and third harmonics. The useful energy range is between 5.5 and $9 \mathrm{keV}$, with the lower limit set by harmonic contamination. The harmonic contamination will range between 0.02 and $0.5 \%$.

To optimize our XPCS set up, it is critical that our optics preserve the brightness of the source and can endure its incident power density. The maximum power density of our undulator at the lowest working energy of $5.5 \mathrm{keV}$ is approximately $145 \mathrm{~W} / \mathrm{mm}^{2}$. In the field of XPCS, typical coherent beam sizes are on the order 

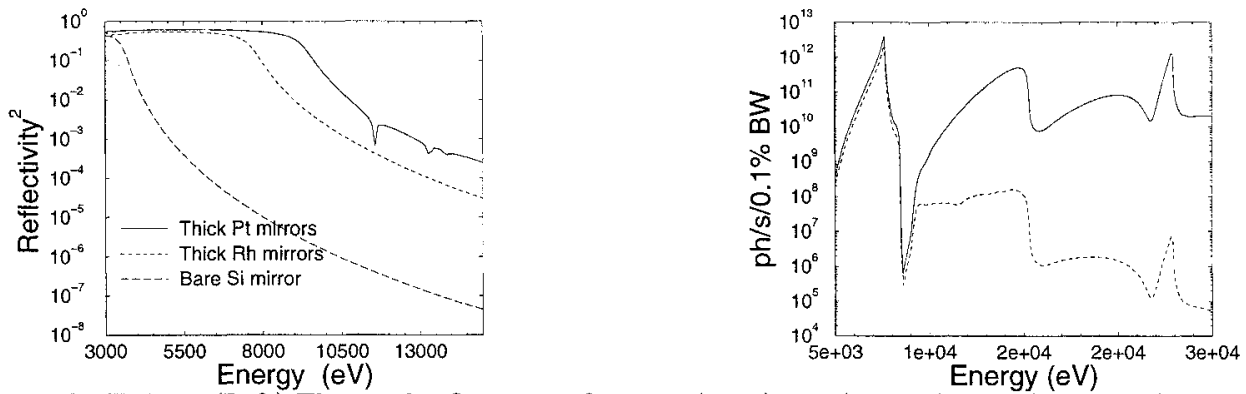

FIGURE 2. (Left) The total reflectivity of two $\mathrm{Pt}$ (solid), Rh (dashed) or Si (long dash) mirrors set at 8.75 mrad. (Right) A typical white undulator beam from the APS (solid) and a doubly reflected "pink" beam (dashed), using the Pt coating.

of 5-10 $\mu \mathrm{m}$, i.e. comparable to the horizontal transverse coherence length of the source. If we accept a small beam of only say $(0.1 \mathrm{~mm})^{2}$ on the mirrors, than the first mirror will only absorb at most $1.45 \mathrm{~W}$ of power, and will still provide an ample beam size. The power density on the mirror is much smaller than that of the white beam due to the large footprint at grazing angle of $0.1 \mathrm{~mm} / 8.75 \mathrm{mrad}=$ $11.4 \mathrm{~mm}$. Given the small power absorbed on the mirrors, no cooling is planned. If one assumes that the power is absorbed on the surface of the mirror only, one can show that the maximum temperature difference between the bulk and beam footprint is

$$
\Delta T=\frac{P}{\pi a \kappa} \ln (4 a / b), \text { for } a>b,
$$

where the range of power $P$ on the first mirror vary from 0.6 to $1.45 \mathrm{~W}$ for the range of useful energies, $b=0.1 \mathrm{~mm}$ is the transverse beam size, $a=11.4 \mathrm{~mm}$ is the beam footprint, and $\kappa=0.131 \mathrm{~W} / \mathrm{mm} / \mathrm{K}$ the thermal conductivity of $\mathrm{Si}[4]$. The temperature rise $\Delta T$ will thus range from 0.8 to $1.9^{\circ} \mathrm{C}$, a small difference.

To preserve the brilliance, the mirror slope errors must be smaller than the angular source size as perceived from the optics, placed $30 \mathrm{~m}$ from the source. The APS is currently running with a $1 \%$ vertical coupling, a horizontal emittance of $7 \mathrm{~nm}-\mathrm{rad}$, and rms source sizes of $\sigma_{x}=321 \mu \mathrm{m}$ and $\sigma_{y}=18.9 \mu \mathrm{m}$ in the horizontal and vertical directions respectively [3]. We have purchased flats with figure errors below approximately $0.4 \mu \mathrm{rad} \mathrm{rms}$ and roughness after coating below $1.8 \AA[5]$. These flats should not reduce the horizontal brightness since the angular source size is above $10 \mu \mathrm{rad}$, but they may reduce the vertical one by up to $20 \%$ since the vertical source size is $0.63 \mu \mathrm{rad}$. Given that the vertical transverse coherence length is a factor 17 larger than the horizontal one, the effective loss of coherent flux for a symmetric aperture will be negligible; nor will the surface roughness reduce the reflectivity significantly.

Each mirror is mounted on the top flange of a UHV tank. The Si flats are 
mounted on standard kinematic mounts (Newport 600A-2R) driven by three vacuum prepared Newfocus picomotors. The angular step size for this mount will be $0.3 \mu \mathrm{rad}$. By driving the three axes simultaneously, one can translate each mirror in and out of the beam by up to $13 \mathrm{~mm}$. Angular and displacement feedback are essential when using picomotors. Tilt sensors (Applied Geomechanics model 755 ) with two tilt axes will record the absolute angular position, while one Linear Variable Differential Transducer (LVDT) (Schaevitz, $250 \mathrm{MHR}$ ) will record the displacement when the three picomotors are used simultaneously. To allow for the selection of a given coating, the mirror kinematic mount is placed on a high precision slide, and driven by a picomotor with linear feedback provided by an additional LVDT (Schaevitz, $500 \mathrm{MHR}$ ). In each of the tanks, a YAG scintilator screen can be inserted to observe the main beam or reflections from the main beam. The visible light fluorescence will be imaged using 1:1 optical collection focused on a TV camera. This will be used for alignment purposes only. Preliminary tests have been performed already, showing that the mirrors preserve the coherence of the source. The assembly will soon be installed in our beamline optical enclosure.

\section{ACKNOWLEDGMENTS}

E.D. acknowledges the support of the Natural Science and Engineering Research Council of Canada. We acknowledge support under NSF Grant No. DMR 9214220, DOE Support through Howard University and through the University of Michigan under grant No. DE-FG02-99ER45743, and the donors of the Petroleum Research Fund, administered by the American Chemical Society under Grant ACSPRF No 32105-AC7. Use of the Advanced Photon Source was supported by the U.S. Department of Energy, Basic Energy Sciences, Office of Energy Research, under Contract No. W-31-109-ENG-38.

\section{REFERENCES}

1. Dierker, S., Pindak, R., Fleming, R., Robinson, I., and Berman, L., Phys. Rev. Lett. 75, 449-452 (1995).

2. del Rio, M. S., and Dejus, R. J., "XOP: A Multiplatform Graphical User Interface for Synchrotron Radiation Spectral and Optics Calculations", in Materials, Manufacturing, and Measurements for Synchrotron Radiation Mirrors, Proceedings of the SPIE, The International Society for Optical Engineering, 1997 vol. 3152 pp. 148-157.

3. Borland, M., Emery, L., Lumpkin, A., Sereno, N., and Yang, B., in Proceedings of the 6th European Particle Accelerator Conference (EPAC-98) Stockholm Sweden: Institute of Physics Publishing, 1998 Vol. 2 p.1556

4. Kutateladze, S., Fundamentals of Heat Transfer, New York: Academic Press, 1963.

5. The Si substrates are $25 \mathrm{~mm} \times 50 \mathrm{~mm} \times 7 \mathrm{~mm}$. Their vertical acceptance is $0.44 \mathrm{~mm}$ at $8.75 \mathrm{mrad}$, and they were purchased from General Optics, 554 Flinn Ave, Moorpark, CA 93021, (805) 529-3324. 